\title{
Robust Full Duplex Radio Link
}

\section{Dinesh Bharadia Stanford University dineshb@stanford.edu}

\author{
Kiran Raj Joshi \\ Stanford University \\ krjoshi@stanford.edu
}

\author{
Sachin Katti \\ Stanford University \\ skatti@stanford.edu
}

\begin{abstract}
This paper presents demonstration of a real-time full duplex pointto-point link, where transmission and reception occurs in the same spectrum band simultaneously between a pair of full-duplex radios. This demo first builds a full duplex radio by implementing selfinterference cancellation technique on top of a traditional half duplex radio architecture. We then establish a point-to-point link using a pair of these radios that can transmit and receive OFDM packets. By changing the environmental conditions around the full-duplex radios we then demonstrate the robustness of the self-interference cancellation to adapt to the changing environment.
\end{abstract}

\section{Categories and Subject Descriptors}

C.2.1 [Computer Communication Networks]: Network Architecture and Design-Wireless communication

Keywords: Full Duplex; Interference Cancellation; Non-linear Cancellation

\section{INTRODUCTION}

Full duplex radios have huge advantage over their half duplex counterparts. Several work on full duplex radios [5, 3, 7, 6, 4, 8] have proposed various hardware and algorithmic techniques to achieve different level of self-interference cancellation required for realization of full duplex radios. Yet, these works have failed to reduce the ingrained skepticism of both academia and industry alike regarding the feasibility of implementing these techniques and their adaptability to dynamic environmental conditions. This demo has two fold objectives; first, we implement a working full duplex radio using the architecture proposed in [5]. We then build full duplex radio link by using a pair of these full duplex radios and demonstrate that they can adapt to the changing environmental conditions around them [5].

In [5] they have shown that in order to achieve full duplex communication with regulatory power limit of existing half duplex WiFi radios, $110 \mathrm{~dB}$ of self-interference cancellation is required. They have achieved this level of cancellation in two stages by designing analog cancellation and digital cancellation filters. After applying these two cancellations, the residue of the self-interference signal goes below the noise floor which enables the receiver to listen to the weak received signal. We demonstrate the cancellation of self-interference all the way to the noise floor by implementing analog and digital cancellation on WARP software radios and a host PC.

Using two such full duplex radios we build a full duplex pointto-point link, where each full duplex radio transmits data to and re-

Permission to make digital or hard copies of part or all of this work for personal or classroom use is granted without fee provided that copies are not made or distributed for profit or commercial advantage, and that copies bear this notice and the full citation on the first page. Copyrights for third-party components of this work must be honored. For all other uses, contact the owner/author(s). Copyright is held by the author/owner(s).

SIGCOMM'14, August 17-22, 2014, Chicago, IL, USA.

ACM 978-1-4503-2836-4/14/08.

http://dx.doi.org/10.1145/2619239.2631425 ceives data from the other full duplex radio via the established link In order to achieve stable full duplex link, it is necessary to achieve cancellation which adapts quickly to the changing environment. This paper demonstrates dynamically adaptive cancellation system, which achieves robust full duplex link even when the environmental condition changes frequently. In the rest of this paper, we first describe the implementation of the cancellation techniques used in our full duplex radio prototype. We then describe the full duplex point-to-point link.

\section{ADAPTIVE CANCELLATION}

Let us see what are the major constituents of the self-interference signals that needs to be canceled to enable full duplex communication. In Figure $1 \mathrm{a}$. $\mathrm{T}$ is the signal that is fed to the antenna for transmission, part of this signal is reflected back from the environment and a part is reflected from the antenna itself, and part of this signal leaks directly to the receive path via spurious path in the circulators. These reflected and leaked transmitted signal denoted by $a T$ in figure 1 a are typically $15 \mathrm{~dB}$ smaller that the signal $T$, therefore we still have to cancel $95 \mathrm{~dB}$ of the self-interference signal. The signal $T$ itself is composed of three parts as given in [5], first is linear form of the transmitted digital data that can be modeled, and the second is the non-linear distortion of the transmitted digital data that can be modeled, third is the transmitter noise which cannot be modeled (as noise is random). Since the transmit noise cannot be modeled in digital, the only way to cancel its effect is to take a copy of it and then cancel it from the received signal. This is best done in analog domain, and as given in [5] we need cancellation of the order of $70 \mathrm{~dB}$ to eliminate all the self-interference due to the transmitter noise.

In addition to canceling the effect of the transmitter noise, the ana$\log$ cancellation also cancels the effect of the environmental reflection from the near-field region of the antenna, as well as the reflection from the antenna itself arising due to impedance mismatch. After the analog cancellation, we are left with the residual signal $i T$, which consists of contribution from the linear and non-linear distortions due to power amplifier, mixer, and other analog components in the transmit chain as well as the contribution from the reflections coming from the far-field region of the antenna. This residual component is modeled adaptively using WiFi preamble to train the digital taps, which can then eliminate this residual signal.

In a typical radio operation, the environmental conditions in the near-field of the antenna is usually stable and does not vary that much over time. Whereas the far field region can experience very rapid changes, mainly due to the movement of persons in the surrounding. Because of this the taps used in analog cancellation of the full-duplex radio remains fairly constant for a given environment in which the radio is located, and consequently slower adaptation of these taps is sufficient. Since the far-field region of the antenna changes frequently, the taps used in the digital cancellation needs to be adjusted more frequently.

In order to demonstrate a dynamic robust full duplex radio, we have build the analog cancellation circuits and digital cancellation using WARP radios [2] as shown in figure $1 \mathrm{~b}$ We have accompa- 


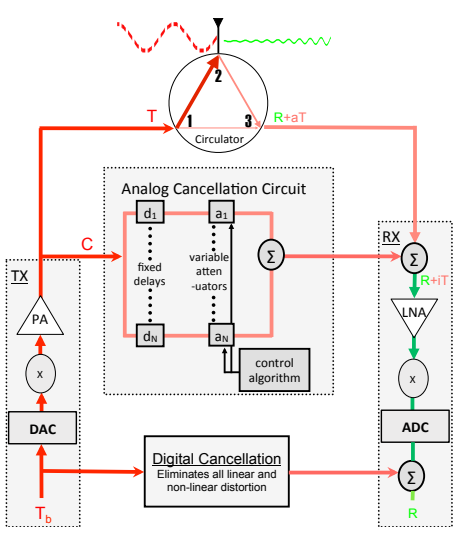

(a) Full duplex radio architecture

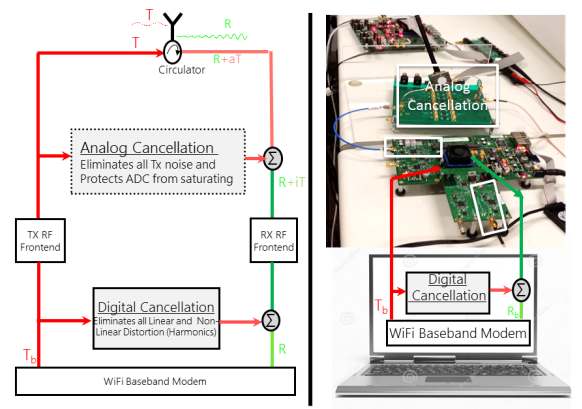

(b) Full duplex radio implementation

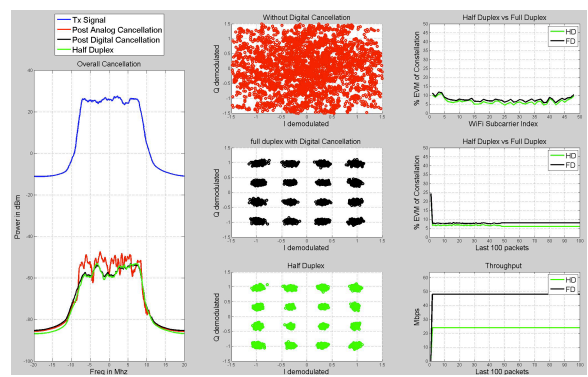

(c) User interface showing performance metric of full duplex link

Figure 1: Demo of robust full duplex Link

nied video of this demonstration in [1]. We see that even when the environment around the full duplex radio is not changing, the taps of the digital cancellation filters are frequently changing, to adapt to the changing condition in the far-field due to movement of people. The taps for analog cancellation remains fairly fixed, because the near field region and the reflection due to the mismatch are not varying over time. To demonstrate that the analog cancellation adapts to the changes in the near-field region, we introduce a metallic reflector very close to the antenna. This reflection adds strong selfinterference in the near-field region which is then compensated for by adjusting the analog taps. We then move the reflector away, which will again cause the change in the near-field region of the antenna and the taps used for the analog cancellation adjusts back to the values that were there before the introduction of the metallic reflector.

\section{POINT TO POINT FULL DUPLEX LINK}

In this section we demonstrate the application of the full duplex radios by creating a full duplex point-to-point communication link. The setup for this link consists of two full-duplex radios that are trying to communicate with each other at the same time on the same spectrum (band). We implement standard 802.11n PHY based WiFi Modem for this demonstration. Figure 1c shows the user interface of the demo with various signals and evaluation metric for one of the radios. In order to compare the performance of the full duplex link to that of the half-duplex link, we alternate the operation between the full-duplex and half-duplex mode for every alternate packet and show the results together. First column in 1c shows the self-interference signals at different stages of the cancellation and the weak received signal after the self-interference cancellation. In the full-duplex mode after the self-interference cancellation, we want the received signal (black) to look exactly like the signal you would have received in the half duplex mode (green). The similarity of these two signals in the attached video indicates that the full-duplex link is operational.

The quality of a link can be visually evaluated by plotting the constellation of the received signal after demodulation. The second column of figure $1 \mathrm{c}$ shows the constellation at various stages of the full duplex link as well as that of the half duplex link. The top plot shows the constellation after only analog cancellation, here the constellation appears as a single blob instead of the 16 constellation points that was used for this demo. The middle plot shows the constellation after both the analog and the digital cancellations and we can clearly see that all the constellation points are tightly grouped around the true points, this tightness indicates the quality of the link. The bot- tom plot shows the constellation for the half duplex link and it looks very close to the full-duplex constellation points, which is yet another confirmation that the full-duplex link is operational.

A more quantitative metric to gauge the quality of a link is to measure \% Error Vector Magnitude (EVM) of the constellation after demodulation. EVM measures the deviation of the constellation points in the received signals from the true constellation points. Therefore smaller the \% EVM for a link higher is the quality of that link. The third column of the user interface shown in figure 1c plots the EVM of the links. As we can see the full-duplex link is slightly degraded compared to the half-duplex link, there is about $2 \%$ difference between the EVM of these two links. As reported in [5], the noise floor after the self-interference cancellation is roughly $1 \mathrm{~dB}$ more than the noise floor of a half-duplex radio, the difference in EVM is due to this marginal increase in the noise floor of the full duplex radio. The top plot show the EVM for each of the subcarriers and the middle plot shows the EVM of each packet for the last 100 packets. The occasional increase in EVM for full duplex link is due to sudden introduction of a reflector close to the radio 1. The taps in analog cancellation takes some time to adapt to this new change and hence the EVM increases momentarily, but once the taps have adapted the link quality increases again. This momentary degradation is an artifact of the current implementation and can be improved upon by performing adaptation using faster hardware support. Finally, the bottom plot shows the near doubling of the throughput via full-duplex link compared to its half-duplex counterpart.

\section{REFERENCES}

[1] Robust Full-duplex Radio link. https://vimeo.com/98308615

[2] WARP Project. http://warpproject.org

[3] E. Aryafar, M. A. Khojastepour, K. Sundaresan, S. Rangarajan, and M. Chiang. Midu: enabling mimo full duplex. In Proceedings of the 18th annual international conference on Mobile computing and networking, Mobicom '12, pages 257-268, New York, NY, USA, 2012. ACM.

[4] D. Bharadia and S. Katti. Full duplex mimo radios. In 11th USENIX Symposium on Networked Systems Design and Implementation (NSDI 14), pages 359-372, Seattle, WA, Apr. 2014. USENIX Association.

[5] D. Bharadia, E. McMilin, and S. Katti. Full duplex radios. In Proceedings of the ACM SIGCOMM 2013 conference on SIGCOMM, SIGCOMM '13, pages 375-386, New York, NY, USA, 2013. ACM.

[6] M. Duarte, C. Dick, and A. Sabharwal. Experiment-driven characterization of full-duplex wireless systems. CoRR, abs/1107.1276, 2011.

[7] Y. Hua, P. Liang, Y. Ma, A. Cirik, and Q. Gao. A method for broadband full-duplex mimo radio. Signal Processing Letters, IEEE, 19(12):793 -796, dec. 2012.

[8] M. Knox. Single antenna full duplex communications using a common carrier. In Wireless and Microwave Technology Conference (WAMICON), 2012 IEEE 13th Annual, pages 1-6, 2012. 